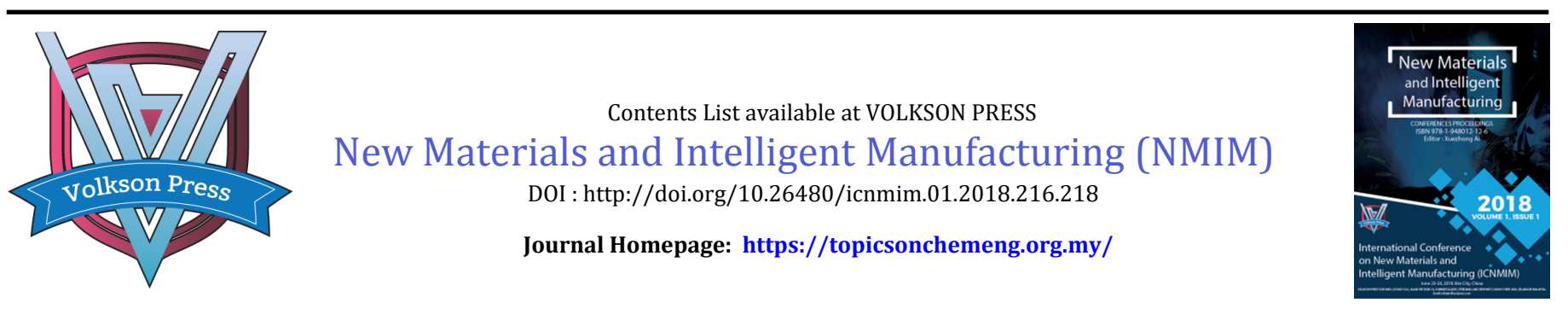

ISBN: 978-1-948012-12-6

\title{
DESIGN AND APPLICATION OF WIRELESS REMOTE TEMPERATURE MONITORING SYSTEM FOR THE BULK GRAIN
}

\author{
Lu Haidong ${ }^{1}$, Wang Xuejing ${ }^{2}$, CaoYubo ${ }^{1}$ \\ ${ }^{1}$ School of Information and Control Engineering, Jilin Institute of Chemical Technology, Jilin 132022 \\ 2PetroChina Jilin Petrochemical Company Synthetic Resin Factory, Jilin City 132021 \\ *Corresponding Author Email:
}

This is an open access article distributed under the Creative Commons Attribution License, which permits unrestricted use, distribution, and reproduction in any medium, provided the original work is properly cited

\section{ARTICLE DETAILS}

\section{Article History:}

Received 26 June 2018 Accepted 2 July 2018

Available online 1 August 2018

\section{ABSTRACT}

The internal temperature of the grain piles is an important indicator of moldy decay of the grain. Real-time monitoring of changes in the internal temperature of the grain piles is an important task in preventing grain mildew. In order to solve the problems of large-scale monitoring of outdoor bulk grain piles and difficulty in cable deployment, a wireless remote temperature monitoring system for bulk grain was designed. The field data collection station was powered by batteries, and a low-power working mode was designed and optimized. The system is of no wiring, low power consumption and easy maintenance, etc., The wireless communication between the host monitoring system and the terminal adopts a simplified Modbus command, which further reduces the wireless communication power consumption of the terminal. This system has achieved good results in practical applications.

\section{KEYWORDS}

Temperature monitoring system, bulk grain, low power consumption, wireless.

\section{INTRODUCTION}

In the course of grain collection and storage, the grain may mildew caused by temperature increase due to such factors as grain ripeness, moisture and impurities. Therefore, the timely and accurate monitoring of the internal temperature of the granaries (reactors) to achieve early prevention of grain mildew is an important issue, which is faced by grain storage, grain processing and storage enterprises at all levels [1].

Under normal circumstances, grain collection and storage enterprises are subject to conditions such as production plans and site area. In the harvesting season of autumn and winter, it is difficult to dry and store the acquired food within a short period of time. A large amount of grain is only stored outdoors in the open air for meeting winter production tasks [2]. At present, most of the grain temperature monitoring products are limited by conditions such as power supply, signal transmission or installation, and cannot meet the requirements for automatic monitoring of the temperature for outdoor bulk grain. The traditional temperature measurement method is generally to pre-embed the temperature measurement cable into the grain pile, and then manually conduct inspection and copying manually.

Under the conditions of grain accumulation, the area of the grain piles is huge and the surface is soft. In the northern areas, there is often snow cover, which poses great difficulties and hidden dangers to human inspection work [3]. The wireless food grain temperature monitoring system is designed and developed to solve this problem. It does not require cables to be laid. Instead of changing the existing food storage and management methods, it uses battery-powered, wirelessly delivered grain reactors. The layer temperature signal is transmitted to the central control room to realize real-time monitoring of the internal temperature of the grain reactor [4].

\subsection{System Structural Composition}

The wireless grain temperature monitoring system consists of a monitoring computer, a wireless communication host, a terminal (including a temperature signal acquisition function), and a temperature measurement cable [5]. The system structure consists of the components shown in Figure 1.

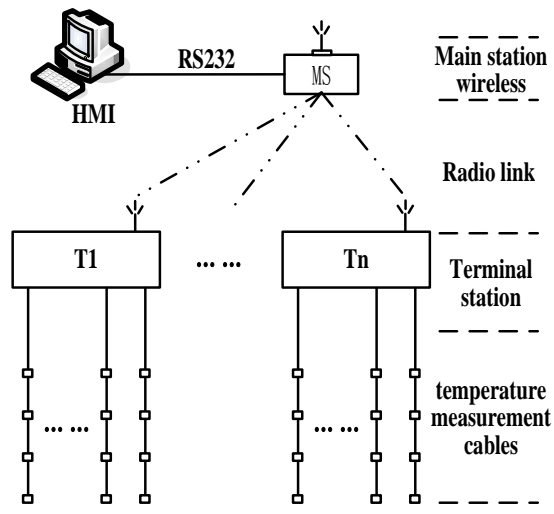

Figure 1: Structure figure of monitoring system

A monitoring computer (HMI) is set in the central control room to display the real-time data of different grain layers in different areas of the grain pile. When the temperature rises above the preset limit, an alarm is started to prompt the staff to carry out food guidance, lighting and ventilation. At the same time, the display interface is published via the Web. The temperature of the grain reactor can be monitored on any computer in the factory-level LAN, and the built-in database can also be used to query historical temperature data (displayed as a historical trend curve).

Between the wireless communication host and each terminal, the data transmission is realized through the wireless RF signal, and the data collected by each terminal is received and sent to the monitoring computer. The communication between the host computer and the monitoring computer adopts 232 connection mode and supports the standard 
Modbus communication protocol. Wireless communication is used between the host computer and the terminal, and a new communication protocol is designed to meet the requirement of saving power and improving radio utilization of the terminal [5].

Since the terminal uses a low-temperature-resistant ordinary battery as a working power source, the circuit itself is designed with low-power consumption and the wireless data communication is optimized as well in order to ensure that the battery satisfies the power supply of the terminal during a grain storage period (generally 6 months), and minimize power consumption and protect battery power. Terminals are generally placed directly on the surface of grain piles, and it is difficult to route cables. Therefore, batteries are used as the working power supply for the terminal, and the 80C51 low-power micro-controller is used as the core to integrate data acquisition, wireless communication, and power saving management functions.

All electronic devices and circuits of the terminals adopt low-temperatureresistant design and can work continuously and stably at $-40^{\circ} \mathrm{C}$. At the same time, considering that the temperature measurement system does not require high real-time performance, a single-chip dormancy management circuit has been designed in order to meet the system energy-saving requirements. The terminal will start every 20 minutes, and most of the time will be in a dormant state, which will reduce power consumption of the system and facilitate system miniaturization and onsite installation.

Each terminal can be connected with 8 temperature measuring cables. 4 temperature measuring points are distributed at a intervals of 1.5 meters per cable. Stratified temperature measurement can be realized for grain piles with a height of 6 to 8 meters. At the same time, the temperature measuring cable passes through 20 meters (The extendable leads are connected to the terminal, so that each terminal can achieve temperature stratification detection in the $40 \times 40 \mathrm{~m}^{2}$ area of the grain pile. Each system can be expanded to the maximum of 40 terminals, real-time monitoring of the internal temperature can be realized for a grain reactor with maximum area of $2000 \mathrm{~m} 2$.

\section{WIRELESS DATA TRANSMISSION AND MANAGEMENT}

\subsection{Wireless communication work flow}

The biggest feature of this system is that the terminal power supply is powered by an ordinary low-temperature high-energy dry battery. It has features such as light weight, low cost and easy to use. It is suitable for outdoor inconvenient wiring applications. The host is placed indoors without having to consider power supply problems [6]. In response to this feature, the system designs a wireless data transceiver mechanism that uses the terminal as the active side and the host as the slave side. The communication system work flow is shown in Figure 2.
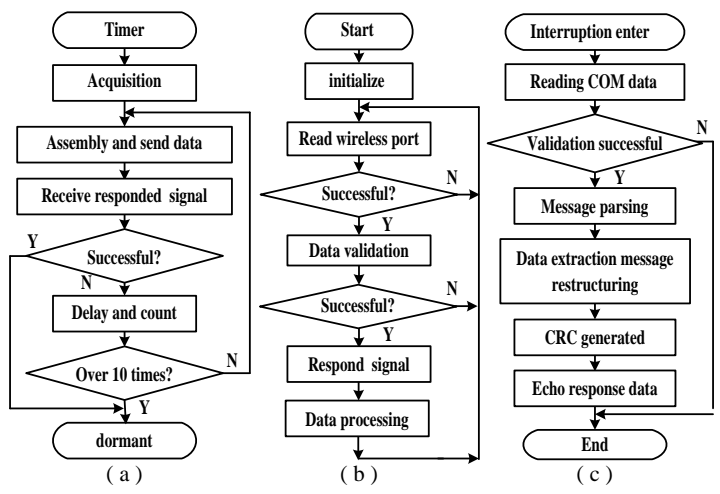

Figure 2: Flow diagram of program

Figure 2(a) is the working flow of the terminal. Under the timing circuit control, the terminal runs every 30 minutes. After the terminal is started, the temperature signal and the battery voltage signal are collected and converted. Then the data frame is assembled and transmitted by the wireless port according to the predetermined format. After receiving the frame of the terminal, the host will reply and confirm. After receiving the acknowledgement, the transmitter will launch again after a random short delay if no confirmation data is received. After the task is completed, reset the timer circuit and go into hibernation again.

Figure 2(b) is the work flow of communication between host and terminal. The host is placed in the central control room. It is not necessary to consider the power supply and wiring problem. The wireless communication program uses the query mode to directly listen to the wireless port, the data is inspected immediately and a reply should be made to confirm when receiving the data of a terminal, and then data storage is carried out according to the terminal number attached in message parsing, and then listen to the radio port again and wait for the next data transfer. The host and the monitoring computer communicate in real time through the serial port and the standard Modbus protocol, and its work flow is shown in figure 2 (c).

\subsection{Improved Modbus communication protocol}

In the wireless communication process, the terminal serves as the active part of the data transmission process, involving the transmission of data frames and the reception of the confirmation frame. Therefore, the wireless communication between the terminal and the host is simplified on the basis of the Modbus protocol. The specific data format is shown in Figure 3.

\begin{tabular}{|c|c|c|c|c|c|}
\hline Data frame & 03 & $\mathrm{XX}$ & & D33 & $\overline{\mathrm{CRC}}$ \\
\hline Respond data & 03 & $\mathrm{XX}$ & 66 & $\mathrm{CRC}$ & \\
\hline
\end{tabular}

Figure 3: Data format of communication

"03" in Figure 3 is the function code (byte), which means the data frame sent by the terminal to the host and the response frame sent by the host to the terminal, "66" in the response frame is the number of bytes received by the host, $\mathrm{XX}$ is the terminal number (byte), corresponding to the actual number of the terminal, "D01 D33" is the data area, a total of 33 words, used to store the 32 temperature values of the 8 temperature measuring cables connected to the terminal and battery voltage value. In order to improve the accuracy of numerical display, the value of $\mathrm{D}$ zone is signal data of input channel. CRC is the check code (double byte). When the terminal receives an acknowledgment frame and the check code is incorrect or fails to receive the acknowledgment frame within the specified time, the terminal will randomly delay and then restart the communication process. The host adopts the polling mode to listen to the radio port. When the data received is verified error, it returns and continues listening.

The terminal is waken up once every 20 minutes and is in radio silence during dominant. This operation mode can significantly reduce the power consumption of the terminal and save radio resources. During the communication process, the terminal served as the active party for wireless data transmission control the wireless transmission process by a random delay to adapt and adjust automatically the wake-up time of each terminal, thereby the wireless channel conflicts between the various communication nodes are avoided and isolated.

\section{MAIN FUNCTION OF SYSTEM}

\subsection{Real-time data display}

The monitoring system has the functions of real-time data display, historical trend query, grain temperature alarm and battery power monitoring. The display of each terminal can be switched by the terminal selection button at any time. The upper part of the screen shows 32 realtime temperature data of the 8 temperature measuring cables connected to the terminal and real-time battery voltage values. The operator can timely control the internal temperature changes of the grain pile according to the data displayed in the screen. they can quickly analyze and judge the metamorphosis of grain mildew such as abnormal temperature increase according to the temperature variation curves of different grain layers [7]. Compared with the manual inspection mode, the real-time data display is greatly improved, and work efficiency is improved and the labor intensity of workers is also greatly reduced. Extension temperature monitoring screen is shown in Figure 4.

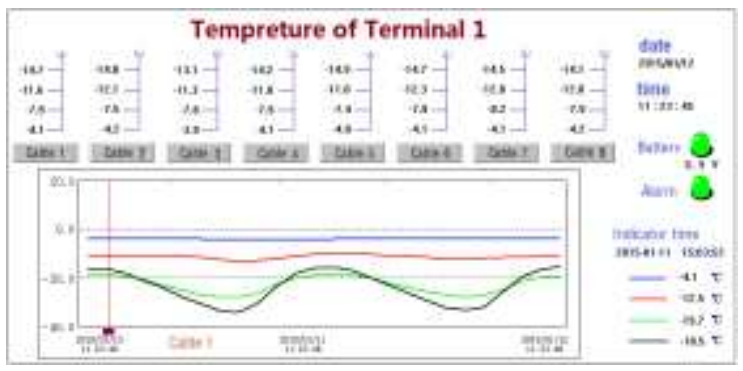

Figure 4: HMI of monitoring system 


\subsection{Historical enquiry function}

The lower half of the screen shows the historical temperature trend curve for 4 temperature measurement points per cable. By clicking on the temperature measurement cable number in the real-time data area, any of the 8 temperature measurement cables can be switched. In the historical curve, it can be intuitively understood that the temperature and its the trend of each grain layer is influenced by the outdoor temperature. The time and temperature values corresponding to the current position of the historical trend tow bar are displayed on the right side of the screen. the temperature data of different time spans or any time interval can be queried through the button at the bottom of the screen. The historical temperature changes of the grain reactor can be easily checked from the trend curve, which is helpful for reference and comparative analysis of grain storage and mildew data.

\subsection{Grain temperature alarm function}

In order to detect more effectively and prevent grain from mildew, the system uses a combination of over-temperature and warming alarms. When the measuring point data exceeds the upper temperature limit, the system alarms. At the same time, since the grain temperature values of each layer are not consistent, the upper temperature alarm value is not easy to determine. Therefore, the system has designed a warming alarm function to compare the temperature measurement data with the outdoor temperature. If the grain temperature increase trend is greater than the outdoor temperature increase trend upper limit, it is determined that the temperature measurement point is an abnormal temperature rise, and the operator is notified to dispose by an alarm signal. This alarm method improves effectively the inspection of grain mildew and provides strong support for ensuring food security storage.

\subsection{Battery power alarm function}

Because the site does not have the wiring conditions, the terminal adopts ordinary low-temperature battery-powered battery with design life of $6 \sim 8$ months (a complete accumulation of grain storage period is generally not more than 6 months). Terminals are placed on the surface of the outdoor grain piles, and the working conditions are rather poor. To avoid the batteries from losing the power supply to the terminals under extreme conditions, the power supply must be known in a timely manner. When the terminal starts, the temperature and battery voltage signals are collected and transmitted to the host computer. The monitoring interface displays battery voltage data in real time and alarms. When the battery voltage drops below 3 volts, the terminal is in danger of shutdown. At this time, the system performs a battery alarm and prompts the operator to replace the battery.

\section{CONCLUSIONS}

Grain temperature testing is an important task to prevent mildew during grain storage and ensure food quality and safety. In this paper, based on the particularity of outdoor grain storage and the shortage of existing grain temperature monitoring products, a wireless grain temperature monitoring system based on battery-powered and wireless transmission is designed. The system adopts the power-saving mode in the terminal design and the power supply problem is effectively solved. Coordination and management of wireless communication based on the terminal are realized by the improved Modbus protocol, so the signal collision problem of multiple communication nodes is avoided. The system has been applied in a number of grain deep processing enterprises and obtains praise from users, various indicators meet the design requirements, and it has the advantages of high reliability, low investment, small size, flexible configuration, simple operation and convenient maintenance, and is suitable for all types of grain processing and warehousing companies. After the system is put into operation, it can remarkedly reduce the labor intensity of workers, achieve the rapid and accurate monitoring of grain temperature, effectively reduced the rate of bad grain, and also reduce the work of dumping and turning over of the grain in the past for concerning about the increase of grain temperature.

\section{REFERENCES}

[1] Yi, J., Qinhua, L., Xuebo, C. 2013. Empty control temperature grain storage technology application [J]. Grain and oil storage science and technology communication, (5), 18-21.

[2] Wencheng, W. 2010. Design of granary multipoint temperature monitoring system based on single chip [J]. Journal of Agricultural Mechanization Research, 32 (7), 7-8.

[3] Shengquan, Z., Zhaohong, X., Zhe, M. 2002. Grain depot temperature centralized monitoring system of new technology [J]. Chinese Journal of Scientific Instrument, (2), 15-16.

[4] Tong, Z., Junjie, S., Le, X. 2014. WebGL technology application in the food temperature monitoring [J]. Journal of Henan University of Technology (Natural Science Edition), 35 (1), 96-99.

[5] Kasim, S., Omar, N.A., Akbar, N.S.M., Hassan, R., Jabar, M.A. 2017. Comparison Semantic Similarity Approach Using Biomedical Domain Dataset. Acta Electronica Malaysia, 1 (2), 1-4.

[6] Abdullah, N.A., Rashid, N.M. 2017. Acquiring Cybercrime Evidence on Mobile Global Positioning System (Gps): Review. Acta Electronica Malaysia, 1 (2), 17-19.

[7] Zamanian, P., Kasiri, M. 2018. Investigation of Stage Photography In Jee Lee's Works And Comparing them With The Works of Sandy Skoglund. Acta Electronica Malaysia, 2 (1), 01-06. 